\title{
The Treatment of Recurrent Trichiasis with Argon Laser Photocoagulation
}

\author{
K. W. SHARIF, A. F. A. ARAFAT, W. C. WYKES \\ Glasgow
}

\begin{abstract}
Summary
We report our experience with the use of Argon laser photocoagulation for the treatment of recurrent trichiasis. A total of 28 lids (of 21 patients) with aberrant lashes were treated in our study. Each lash was treated with a beam of 50-200 micron (spot size), $0.2 \mathrm{~s}$ (time), and 1-1.2 Watt (power). Neither infiltration nor topical anaesthesia was used. The mean follow-up time was 6.57 months. Successful treatment with no evidence of recurrence was achieved in $67.9 \%$ of lids after one to two laser sessions. The remaining $32.1 \%$ of the lids required three to four sessions to achieve no recurrence of the condition. There was a significant correlation between the number of aberrant lashes per lid and the number of required laser sessions. Complete healing of the treated areas occurred within six weeks after treatment, with no vascularisation or distortion of the lid margin. Argon laser treatment appears to be a safe and effective alternative to the other recognised methods of therapy in selected cases.
\end{abstract}

Treatment of trichiasis has always been a difficult therapeutic problem. Many modalities of therapy have been tried with variable rates of success. These include epilation after which rubbing lashes invariably recur; electrolysis which has not been fully successful in the past; ${ }^{\prime}$ and cryotherapy with its well reported side effects. ${ }^{2}$ Surgical procedures involving the lid margin are usually reserved for patients with widespread areas of trichiasis. ${ }^{3}$

The use of argon laser photocoagulation for the treatment of trichiasis was first suggested by Berry in $1979 .^{+}$This method of treatment did not receive much attention until recently, when Awan reported successful use of the laser in treating a small number of patients with trichiasis. ${ }^{5}$

The aim of our prospective study was to determine the efficacy of the laser therapy by recruiting a larger number of patients with long term follow-up after treatment. The other objectives of this study were the assessment of any possible side effects of the therapy, and the degree of patients' acceptability to the procedure.

\section{Materials and methods}

Twenty-eight eyelids of 21 patients- 11 males and 10 females-with trichiasis involving the upper lid, lower lid, or both, were recruited for this study. The age of the patients ranged from 50 to 86 years, with a mean age of 67.3 years. The duration of symptoms ranged from six months to six years. Each patient had had repeated epilation and/or recurrence of rubbing lashes after electrolysis therapy. Patients with more than 10 rubbing lashes per eyelid and those who had associated entropion were 


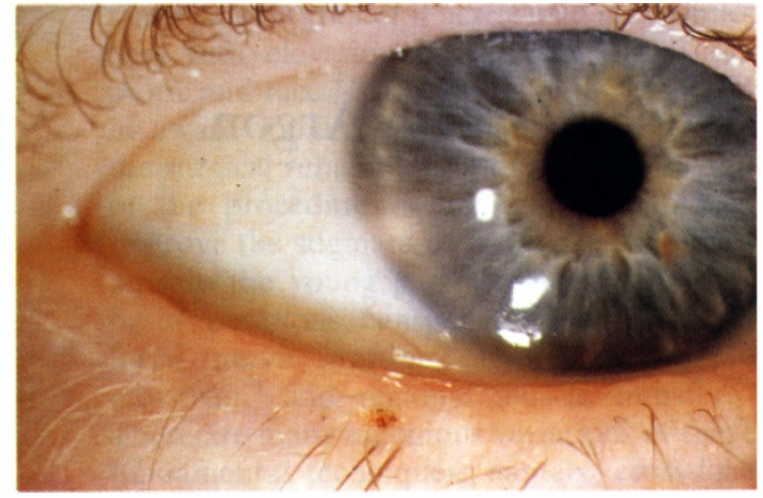

Fig. 1. The initial application of the laser- 150 microns) has produced a crater at the base of the aberrant eyelash.

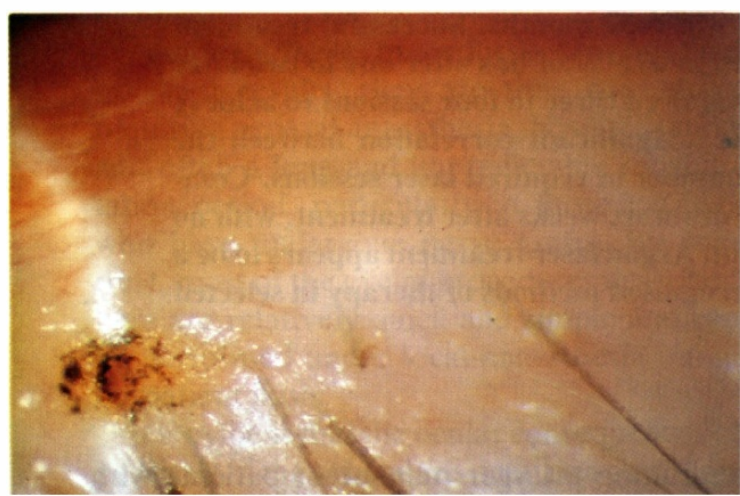

Fig. 2. High magnification slit lamp photograph demonstrates further deepening of the initial crater-by 200 micron successive applications-to ablate the whole hair follicle.

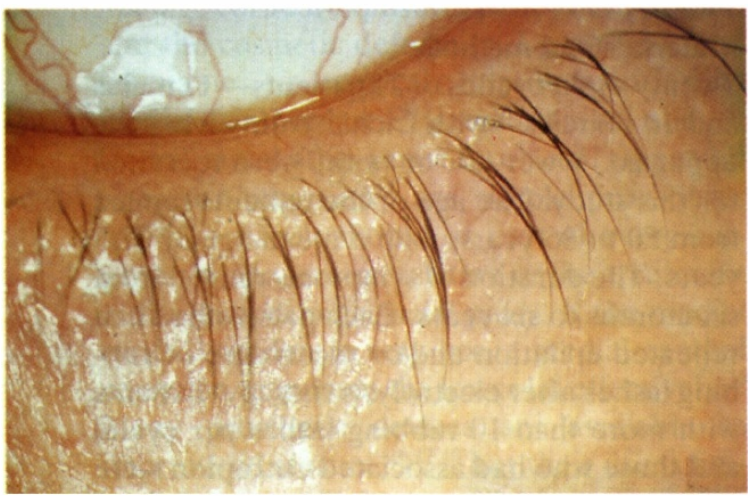

Fig. 3. The lid margin of the patient shown in Figure 2 , with no evidence of recurrence two months following laser therapy. Note the complete recovery of the treated area with no distortion or vascularisation. excluded from this study and referred for other methods of treatment.

The procedure was explained to the patient and an informed consent obtained. After conducting an ophthalmic examination, which included assessment of visual acuity and detailed examination of the eyelids to document the exact site of the aberrant lashes and to determine the presence or absence of any co-existing lid scarring, neovascularisation or entropion, the patient was positioned at the slit lamp of the laser unit and the eyelid was rotated slightly outwards to align the laser beam with the axis of the root of the rubbing lash. The patient was then asked to look away from the direction of the eyelid being treated so that no part of the cornea was visible in the treatment field. The laser controls were set to a spot size of 50 microns, a time exposure of $0.2 \mathrm{~s}$ and a power of 1-1.2 watts. The beam was focused at the root of each cilium so that its path was co-axial with the subcutaneous portion of the eyelash. The initial application of the laser divided the shaft of the lash at its base and created a crater (Fig. 1). This was then deepened to destroy the whole lash follicle with an increased spot size of 200 microns (Fig. 2). This usually took an average of 20 applications to complete. All maldirected lashes were treated in the same way. No anaesthetic was used for the procedure. At the conclusion of the laser treatment the patient was discharged on topical antibiotic ointment to be applied twice daily for one week. The whole procedure was quick, taking about one minute per lash, and the patients felt only a slight smarting sensation.

The patient's first follow up appointment was scheduled for six weeks after therapy to allow any remaining ingrowing lashes, e.g. those which had been epilated just before the initial treatment session, to grow enough to be clearly visualised. These were then treated. At each follow up appointment the visual acuity was checked and the lids examined as described above. If no rubbing lashes were seen at six weeks, follow up appointments were arranged for three and six months. Patients were advised to report back earlier if they experienced any symptoms suggestive of recurrence. Recurrent lashes were treated with repeat laser therapy following the set protocol. 
Table 1. Causes of trichiasis among the population of the study

Blepharitis

Herpes zoster ophthalmicus

Pemphigoid

Surgical scar (excised basal cell carcinoma)

Idiopathic

\section{Results}

Local inflammatory conditions and pre-existing scarring were identified as the underlying aetiology of rubbing lashes in $38 \%$ of cases (Table I). The upper lid was involved in 13 eyes and the lower lid in 15 eyẹs. This included three eyes in which the upper and lower lids were involved. The numbers of rubbing lashes per lid ranged from one to nine with an average of 4.1 lashes per lid (Table II). The follow-up period after the last session of laser treatment ranged from two to 12 months, with a mean of 6.57 months.

The goal of no recurrence over the follow-up period was eventually achieved in all patients. Table III lists the number of sessions per eyelid required to achieve this result. Over $67 \%$ of the lids were successfully treated with two sessions or less, while four sessions were necessary in only $10.7 \%$ of the treated lids.

The procedure was well tolerated by the patients, and nearly all of them experienced a mild smarting sensation as well as lacrimination during treatment. When lacrimation was excessive it required repeated drying of the lid margin before continuing therapy. The patients, when asked at later appointments, did not report any post treatment problems. In particular there were no visual effects noted and there were no signs of lid scarring or vascularisation secondary to the procedure. All the treated areas had healed completely within six weeks after therapy leaving smooth and even lid margins (Fig. 3).

Table II. Number of rubbing lashes per lid

\begin{tabular}{cc}
\hline Number of lashes & Number of lids \\
\hline 1 & 4 \\
2 & 4 \\
3 & 3 \\
4 & 4 \\
5 & 5 \\
6 & 5 \\
76 & 3 \\
\hline
\end{tabular}

\section{Discussion}

Trichiasis, or acquired posterior orientation of previously normal eyelashes, is a relatively common disorder. If left untreated it can lead to corneal ulceration, vascularisation, hypoethesia and can predispose to infectious keratitis. ${ }^{6}$ This study demonstrates the successful use of argon laser therapy to treat trichiasis without topical or infiltration anaesthesia. The other available modalities for treating this difficult clinical problem are not satisfactory and can be associated with various complications. Electrolysis has a high recurrence rate, it is associated with excessive scarring, and requires infiltration anaesthesia. ${ }^{7}$ Cryotherapy also requires infiltration anaesthesia and may produce many adverse reactions. ${ }^{8.9}$ A thermocouple may be required to monitor the temperature of the treated tissue to achieve better results. ${ }^{6}$ Pigment cells are extremely susceptible to freezing and can be destroyed at a temperature of $-10^{\circ} \mathrm{C}$. ${ }^{10}$ Hair follicles are destroyed at a temperature of $-20^{\circ} \mathrm{C}$, and thus skin depigmentation will nearly always occur if aberrant lashes are to be effectively treated. This complication limits the use of cryotherapy in black patients. ${ }^{11}$ Cryotherapy of the lid margin results in sectorial ablation of all lashes in the frozen area, both normal and abnormal, as it does not discriminate trichiatic lashes. Some tissue shrinkage occurs and this can lead to the formation of a notch in the tissue of the lid margin. Thinning of the lid and, in particular, the lid margin results from the loss of the lash follicles, destruction of many of the meibomian glands, Moll's glands, and the glands of zeis and probably some loss of thickness of the orbiculuris muscle and skin. ${ }^{6}$ Patients who have borderline tear film are noted to be more symptomatic following freezing even though the lashes are ablated; hence it is suggested that freezing of the tarsal glands reduces the integrity of the precorneal tear film. ${ }^{3}$ It has also been suggested that cryotherapy should

Table III. Number of laser sessions required per lid

\begin{tabular}{lcc}
\hline No. of sessions & No. of lids & Percentage \\
\hline 1 & 11 & 39.3 \\
2 & 8 & 28.6 \\
3 & 6 & 21.4 \\
4 & 3 & 10.7 \\
\hline
\end{tabular}


not be used in treating patients with trichiasis secondary to ocular pemphigoid as they can have exacerbation of their disease due to inciting the auto-immune reaction. ${ }^{3}$

Our study confirms that argon laser therapy is a safe and effective alternative to the above mentioned modalities for the treatment of recurrent trichiasis.

Argon laser emits a coherent blue-green light of about $488-515 \mathrm{~nm}$ wavelength. This is absorbed by the melanin pigment of the treated eyelash, and the subsequent conversion of the laser light into heat energy produces the therapuetic thermoablative effect. As the laser is applied under slit-lamp magnification, it provides a selective and accurately controlled ablation of the aberrant lash without affecting the surrounding healthy tissue.

The patients in our series showed no evidence of recurrence of the ingrowing lashes during a mean follow-up time of 6.57 months, after one or more laser sessions. It is important however to note that lids with ten or more ingrowing lashes were not included in our study as other modalities of treatment were considered to be more suitable. $67.9 \%$ of the lids showed no recurrence following one to two laser sessions (Table III). The remaining $32.1 \%$ of the lids, that required three or more sessions, had thick and numerous aberrant cilia (more than five per lid). This significant correlation between the number of aberrant lashes per lid and the number of required treatment sessions to achieve no recurrence $(\mathrm{P}=<0.05)$, can be explained by the observation that in the case of numerous lashes, it was more difficult to apply enough laser burns per lash to allow for deep penetration in a single laser session. Hence, the incomplete destruction of some of the hair follicles and the need for repeated laser therapy. The follicle of an eyelash is about $2 \mathrm{~mm}$ in depth from the lid margin surface. ${ }^{12}$ To achieve successful destruction of the hair follicle, the crater produced by the laser should be deep enough along the axis of the shaft to the treated cilium (Fig. 2).

Although Berry used infiltration anaesthesia prior to laser therapy ${ }^{4}$ and Awan used topical anaesthesia ${ }^{5}$, we found that anaesthesia was not required. None of our patients had any form of anaesthesia before laser application and they all tolerated the session very well with very minimal discomfort. This interesting finding can be attributed to the extremely short duration of laser application, very fast destruction of the tissue and the minuteness of the area affected by each laser application. However, we found that patients who had undergone treatment for more than five aberrant lashes per lid experienced slightly more discomfort than others. This is the same subgroup of patients who required three to four laser sessions to achieve complete cure with no recurrence. Thus, it might be advisable to use infiltration anaesthesia in this particular subgroup, to allow the surgeon to go very deep with laser burns to destroy the follicles in one sitting.

There was no operative or postoperative bleeding in any of the treated eyelids. All the treated areas healed smoothly within six weeks with no distortion of the lid margin or vascularisation (Fig. 3).

The laser therapy, however, had some limitations. It cannot be used as effectively in eyelids with ten or more aberrant lashes; it cannot be applied to bedridden patients, or those with head tremours. It was also difficult to treat very nervous and very young cases who were not co-operative enough to facilitate the precise application of laser.

In conclusion, our study shows that argon laser thermoablation is an effective and safe method for the treatment of recurrent trichiasis with many advantages over other recognised modalities of therapy. The main advantages are; the convenience of treating trichiasis as an outpatient procedure; the treatment session is less time consuming than other methods of therapy; no need for infiltration or topical anaesthesia; precise and selective treatment with no destruction of surrounding healthy tissue or depigmentation of the skin; and the very low incidence of recurrence which can be minimised by repeated laser sessions.

The authors are grateful to Dr J. Williamson, Dr W. M. Doig and Dr P. M. Kyle for allowing us to recruit their patients for this study.

Ethical Committce approval has been granted for our trial. 


\section{References}

${ }^{1}$ Fox, SA: Ophthalmic surgery, 4th ed. New York. Grun Stratton, 1970, 330-5.

2 Sullivan JH, Beard C, Bullock JD: Cryotherapy for treatment in trichiasis. Am J Ophthalmol 197: 82: 117-18.

${ }^{3}$ McCord CP: Surgery of the evelids: in Duane T. $n$ (ed.) Clinical Ophthalmology. Philadelphia. Ha: per \& Row 1987, 5 (5): 1-53.

${ }^{4}$ Berry J: Recurrent trichiasis: treatment with lawer photocoagulation. Ophthalmic Surg 1979. 10: 36-8.

${ }^{5}$ Awan KJ: Argon laser treatment of trichiasis. Ophthalmic Surg 1986, 17: 658-70.

${ }^{6}$ Kaufman HE. McDonnald MB, Barron BA, Waltman SR: The cornea, Churchill Livingstone Inc., New York, 1988, p. 163-167.

${ }^{7}$ Scheie HG and Albert DM: Distichiasis and tri- chiasis, origin and managment. Am J Ophthalmol 1966, 61: 718-20.

"Hecht SD: Cryotherapy of trichiasis with use of the retinal cryoprobe. Ann Ophthalmol 1977, 912: 1501-2.

"Fraunfelder FT and Petursson GJ: The use of liquid nitrogen cryospray for treatment of trichiasis. Ophthalmic Surg 1979, 10: 42.

${ }^{11}$ Collin JRO, Coster DJ, Sullivan JH: Cryosurgery for trichiasis. Trans Ophthalmol Soc UK 1978, 98: $81-3$.

"Collin JRO: Manual of systematic lid surgeryLondon, Churchill-Livingstone, 1983, 25-26.

${ }^{12}$ Duke-Elder S and Wybar KC: Anatomy of the eye. In Duke-Elder S. (ed.); System of Ophthalmology, Vol. 2, St Louis. The CV Mosby Company, 1961, p. 526. 